\title{
Organización didáctica de los materiales tecnológicos: el caso del blog educativo
}

\author{
Fernando Gómez-Gonzalvo, Silvia Cardiel, Julio Guerrero, Elena Pardos-Mainer y \\ Lucía Sagarra-Romero \\ ${ }^{a}$ Universidad San Jorge. Grado en Ciencias de la Actividad Física y el Deporte.
}

\begin{abstract}
In the present work an experience in the use of blogs in the physical activity and sports sciences degree in three subjects of the degree is shown. Two of them of the first course (danza y expresión corporal and sistemática del ejercicio) and one of third course (juegos y deportes alternativos). An inductive type analysis is carried out to extract the categories in which the students, through the comments of the blog, show their perceptions about the use of the blog in university educational contexts.
\end{abstract}

Keywords: blogs, collaborative knowledge, assessment, ICT

\begin{abstract}
Resumen
En el presente trabajo se muestra una experiencia en el uso de blogs en el grado de ciencias de la actividad física y el deporte en tres asignaturas de la titulación. Dos de ellas del primer curso (danza y expresión corporal y sistemática del ejercicio) y una de tercer curso (juegos y deportes alternativos). Se realiza un análisis de tipo inductivo para extraer las categorías en las que el alumnado, a través de los comentarios del blog, muestra sus percepciones sobre el uso del blog en contextos educativos universitarios.
\end{abstract}

Palabras clave: Blogs, aprendizaje cooperativo, evaluación, TIC

El presente trabajo procede del proyecto de innovación educativa El uso de blogs en educación superior. Aproximación al aprendizaje colaborativo, la evaluación formativa y el aprendizaje significativo, financiado por la Universidad Francisco de Vitoria, en la convocatoria 2018. 


\section{Introducción}

La tecnología se posiciona hoy en día como un elemento esencial en la sociedad ya que se ha convertido en un elemento con entidad propia que, además, se ha posicionado como herramienta de comunicación en las sociedades occidentales. Está presente en todos los ámbitos de la sociedad y es considerada uno de los principales ejes entrono a los que se está construyendo la realidad, los cuerpos y el poder (Harari, 2016).

Según algunos autores, el uso de las tecnologías de la información y la comunicación (TIC) en el ámbito educativo ha supuesto una mejora del proceso de enseñanza-aprendizaje por la innovación aportada por estas herramientas y, además, ha supuesto un cambio de paradigma educativo (Marqués y Álvarez, 2014). Esta posición ha sido duramente criticada debido a que la introducción de una herramienta, por sí sola, no es capaz de cambiar el contexto, sino que debe estar integrada en una planificación metodológica y didáctica (Gros y Suárez, 2016). Bates (2009) señala que la utilización de las tecnologías únicamente ha supuesto una traducción al formato digital de las actividades y contenidos que se encuentran en los libros de texto, y mantiene intacta la lógica de la educación: el alumnado debe repetir lo que se realiza en las actividades que se desarrollan en el aula.

La innovación no es un proceso universal, sino que depende de dos factores. El primero de ellos es el contexto donde se realiza y, el otro, es que el elemento que se introduce aporte algo no realizado hasta el momento. Es decir, el uso de una tecnología puede ser innovador únicamente si se introduce en un contexto donde no existía antes y es capaz de lograr un cambio en las dinámicas que se realizan. No obstante, y siguiendo a Sparkes (1992), existen diferentes niveles de cambio educativo. Según este autor, existen tres niveles de cambio, y cada uno de ellos, se caracteriza por lograr un cambio más profundo y complejo en la realidad. El primer nivel de cambio se realiza a la hora de introducir nuevos materiales curriculares; en el segundo nivel se produce un cambio en los estilos de enseñanza y en las dinámicas de clase y; el tercer nivel de cambio, se produce cuando se modifican los sistemas de valores, creencias e ideologías.

Se ha escrito mucho sobre los beneficios e inconvenientes del uso de blogs en todos los niveles educativos, de forma que se acepta como una herramienta válida para favorecer el aprendizaje. Entre los beneficios se ha encontrado que desarrollan las habilidades de lectoescritura, las habilidades sociales y de comunicación, el intercambio de ideas y la participación del alumnado que suele ser menos participativo (Santos y Fernández-Rio, 2012; Sim y Hew, 2010), e incluso, aumenta la toma de responsabilidades del alumnado cuando el blog depende de ellos (Wang y Hsua, 2008). Como inconvenientes se ha encontrado que supone una carga extra de trabajo para alumnado y profesorado, que existen limitaciones técnicas que pueden dificultar el acceso al blog y que se tiene la posibilidad de abandonar el blog si la experiencia resulta negativa (Domingo y Marqués, 2013; Wang, Lin y Liao, 2012).

Como señalan algunos autores, las características de los blogs permiten fomentar espacios de debate, de intercambio de ideas y de interacción entre el alumnado (Coutinho, 2007), pero en la mayoría de casos se utiliza como un complemento de la clase presencial (Aparicio, 2010). Sin embargo, las posibilidades de uso de los blogs van más allá del uso

(cc) BY-NC-ND 2018, Universitat Politècnica de València

Congreso IN-RED (2018) 
como complemento de clase. Antolín, Molina, Villamón, Devís-Devís y Pérez-Samaniego (2011) señalan cuatro usos del blog en función de la participación del alumnado y profesorado:

- Como transmisor de información: el blog se utiliza para colgar información sobre la asignatura por parte del profesorado.

- Abierto a la participación del alumnado a través de los comentarios: Su utilización se centra en que el alumnado debe realizar comentarios a los post propuestos por el profesorado.

- Abierto a la participación del alumnado a través de post: este uso se caracteriza porque además de poder realizar comentarios, el alumnado puede proponer la realización de post propios de forma que éstos colaboran como autores del blog.

- Alumnado como administradores del blog: este uso se caracteriza porque el alumnado administra su propio blog tomando decisiones sobre la configuración del blog, el contenido o la forma de los post.

Tal y como indican estos autores, los diferentes tipos de uso de blog se pueden ordenar en un continuo según la participación del alumnado, desde posturas pasivas hasta la muy activas. De esta forma, el uso del blog como transmisor de información se sitúa como la opción más pasiva del alumnado, en la que únicamente recibe información. El uso del blog en que el alumnado pueda realizar comentarios o entradas supone una opción moderadamente activa puesto que la implicación del alumnado es considerable, pero no afecta a la gestión y organización del blog. Por último, que el alumnado sea el administrador de su propio blog es la opción de uso más activa, no sólo porque debe decidir sobre el contenido o la gestión, sino que también debe responder a los comentarios del resto de compañeros creando situaciones de feedback autorregulado por el alumnado.

Hasta el momento, las propuestas realizadas en torno al uso de blogs en entornos universitarios, han estado integradas dentro de las planificaciones docentes de las asignaturas, pero existen pocas propuestas que hayan tratado de integrar de forma transversal el uso de blogs siguiendo una planificación que implique a varias asignaturas. La intención de la presente propuesta de innovación es que el proceso de uso del blog no quede como una anécdota en una asignatura, sino que esté integrado en el grado de forma que el alumnado vaya asumiendo mayor grado de responsabilidad en su uso.

\section{Objetivos}

Los objetivos que se plantearon para el alumnado a través de esta propuesta innovadora fueron:

\subsection{Objetivos generales:}

- Responder a los nuevos requerimientos educativos mediante el uso de las TIC y de un blog (objetivo 1).

- Integrar el blog educativo como una herramienta transversal de aprendizaje que permita mejorar la adquisición competencial de capacidad para aplicar las TIC en el ámbito de las ciencias de la actividad física y el deporte (objetivo 2). 


\subsection{Objetivos específicos:}

- Utilizar el blog con fines educativos, de difusión o informativos sobre la actividad profesional y/o académica del alumnado (objetivo 3).

- Generar recursos didácticos accesibles, prácticos y de gran utilidad relacionados con contextos específicos de las diferentes asignaturas (objetivo 4).

- Desarrollar las capacidades de búsqueda, selección, síntesis y expresión de la información (objetivo 5).

- Asentar conocimientos de enseñanza-aprendizaje de una forma interactiva y motivante para el alumnado (objetivo 6).

- Compartir ideas y debatir sobre temas relacionados con el campo de la actividad física y el deporte enfocado desde cada asignatura (objetivo 7).

- Favorecer la utilización de una herramienta de trabajo útil que permita la integración, participación activa y socialización del alumnado (objetivo 8).

- Explorar formas de evaluación que impliquen de forma activa al alumnado (objetivo 9).

\section{Desarrollo de la innovación}

Este proyecto se desarrolló en el grado en ciencias de la actividad física y el deporte (CCAFD) de la Universidad San Jorge, abarcando 3 asignaturas de $1^{\mathrm{er}}$ y $3^{\mathrm{er}}$ curso de la titulación.

Tal y como se ha señalado anteriormente, la introducción de los blogs como elementos de innovación ha de tener en cuenta el contexto en el cual se realiza. En este sentido, el grado de CCFAD no contaba con experiencias previas de utilización y puesta en práctica de esta herramienta innovadora en ninguna asignatura desde su implantación en el curso 2011/2012. Consecuentemente, esta es la primera vez que se lleva a cabo el presente proyecto en el contexto de este grado universitario.

El diseño y desarrollo del blog se ha implementado en tres asignaturas del grado en CCAFD: danza y expresión corporal, sistemática del ejercicio y juegos y deportes alternativos.

La asignatura de danza y expresión corporal $\left(1^{\mathrm{er}}\right.$ semestre) y sistemática del movimiento ( $2^{\circ}$ semestre) pertenecen al $1^{\mathrm{er}}$ curso mientras que juegos y deportes alternativos ( $2^{\circ}$ semestre) es una asignatura del $3^{\text {er }}$ curso del grado.

El proyecto tiene la intención de no sobrecargar el trabajo del profesorado y, además, conseguir que el trabajo del alumnado sea público y democrático a exponer qué se está realizando. La presentación a través del blog permite compartir el contenido con el resto de compañeros, y con la población en general, para que estos puedan dar feedback y mejorar el trabajo y añade un componente innovador que pretende aumentar la motivación del alumno ante la tarea. 
En la asignatura de Danza y Expresión corporal estaban matriculados 63 alumnos y alumnas. La participación en el blog se evaluaba con un $10 \%$ de la calificación final y se pedía al alumnado un mínimo de 10 comentarios para su evaluación. El profesorado era el administrador del blog y este se utilizó de forma abierta a la participación del alumnado, de forma que, además de los comentarios a los post que proponía el profesorado podían realizar post de elaboración propia. Los comentarios debían tener una extensión entre 500700 palabras. El alumnado realizó 5 post con temáticas diferentes como el análisis de obras que había visto, la explicación de diversas danzas del mundo, propuestas innovadoras en teatro o la exposición de los trabajos que habían realizado en clase.

El blog se compuso de un total de 16 post en los que se desarrollaban contenidos sobre expresión corporal a través de vídeos, artículos, reflexiones e imágenes. Los temas tratados fueron el gesto y el simbolismo, la construcción musical, el ritmo, la construcción coreográfica, definición de danza y expresión corporal, currículum oficial de educación física y talleres que se realizaron a lo largo del curso. Por último, se realizó un post de reflexión final donde el alumnado expresó su opinión y evaluó el desarrollo del blog. Este post fue utilizado en el análisis de datos.

En la asignatura de sistemática del ejercicio había un total de 65 alumnos y alumnas matriculadas. La participación en el blog se evaluaba con un porcentaje de calificación de $20 \%$. En esta asignatura la tarea que debía realizar el alumnado en el blog era la publicación del trabajo individual. Este consistía en la elaboración de un post relacionado con el tema a trabajar que se asignaba al alumnado. Eran ellos quienes se encargan de aportar contenido al blog de la asignatura. Por lo tanto, se utilizó el blog de forma abierta a la participación del alumnado de forma que ellos eran quienes debía desarrollar el contenido íntegro del blog mientras que la administración quedaba a cargo del profesorado.

Se elaboraron unas directrices que debían seguir para elaborar el post. La primera parte era una introducción, en la que debían investigar brevemente sobre el tema a desarrollar; el desarrollo del trabajo con las propuestas prácticas de distintos ejercicios orientados al tema trabajado y; finalmente, los alumnos debían incorporar las referencias bibliográficas que habían utilizado para desarrollar el post. Entre las directrices dadas al alumnado se les pedía que incluyeran, en la medida de lo posible, material multimedia de autoría propia. Los temas del trabajo del blog se asignaban de manera aleatoria entre el alumnado y se debía presentar el post terminado en la fecha indicada. De esta forma se intentó crear una dinámica en la que cada semana el blog tuviera varias entradas nuevas relacionadas con el trabajo que se realizaba en la clase teórica y respetando la planificación de contenidos, de forma que, se publicaban los trabajos individuales del alumnado en la misma semana en la que se trabajaban esos mismos contenidos en las clases teóricas.

En el caso particular de esta asignatura, el blog está en este momento en sus inicios por lo que todavía no es posible detallar resultados del mismo. No obstante, la impresión por el momento es positiva y se aprecia interés por parte del alumnado en cuanto al trabajo propuesto. Al finalizar el blog se recogerán datos de forma cualitativa para su análisis a través de un último post de autovaloración.

(cc) EY-Nc-No 2018, Universitat Politècnica de València 
Por último, en la asignatura de juegos y deportes alternativos el blog sirve como herramienta para poner en común las sesiones que realiza cada grupo de trabajo sobre un deporte alternativo. En esta asignatura había matriculados un total de 71 alumnos y alumnas. El blog se evaluaba con un $50 \%$ de la calificación del trabajo en grupo que, a su vez, era calificado con el $20 \%$ de la nota final de la asignatura. El profesorado compartía parte de la responsabilidad dejando al alumnado como administrador del blog.

\section{TCHOUKBALL}

\section{HISTORIA DEL TCHOUKBALL}

El Tchoukball fue diseñado por el Dr. Brandt de la Universidad de Génova, haciendo una adaptación del Balonmano con la intención de evitar al máximo las situaciones conflictivas con el jugador o jugadora adversaria. Y posteriormente fue introducido en España por Manuel Martínez Gámez, maestro y licenciado en educación física, especialista en Juegos y Deportes Alternativos y ex-presidente de la Asociación Española de Tchoukball (AETB).

El 28 de julio de 2011 queda registrada por el Ministerio la Asociación Española de Tchoukball. La primera vez que la selección participó en un campeonato internacional fue en Ferrara (Italia), equipo que preparó David García Castro

El 3 de agosto de 2011 se aprueba nuestro ingreso en la FITB (Federación Internacional de Tchoukball).

REGLAMENTO DEL TOUCHKBALL

\section{El Terreno de juego:}

- Dimensiones $40 \times 20$ metros.

- Zona prohibida: radio de 3 metros.

- Líneas (ancho $5 \mathrm{~cm}$ ):

- Líneas laterales.

- Líneas de atrás.

- Línea central.

- Obstáculos separados $1-2$ metros por fuera del campo.

- Marco: fijado al suelo durante el juego.

El balón:

- Categoria masculina: circunferencia de 58 y $60 \mathrm{~cm}$ y peso de 425 y $475 \mathrm{gr}$.

- Categoría femenina: circunferencia de 54 y $56 \mathrm{~cm}$ y peso de 325 y $400 \mathrm{gr}$

- Categoría mixta: usaremos el de la categoría femenina.

(cc) EY-NC-ND 2018, Universitat Politècnica de València 


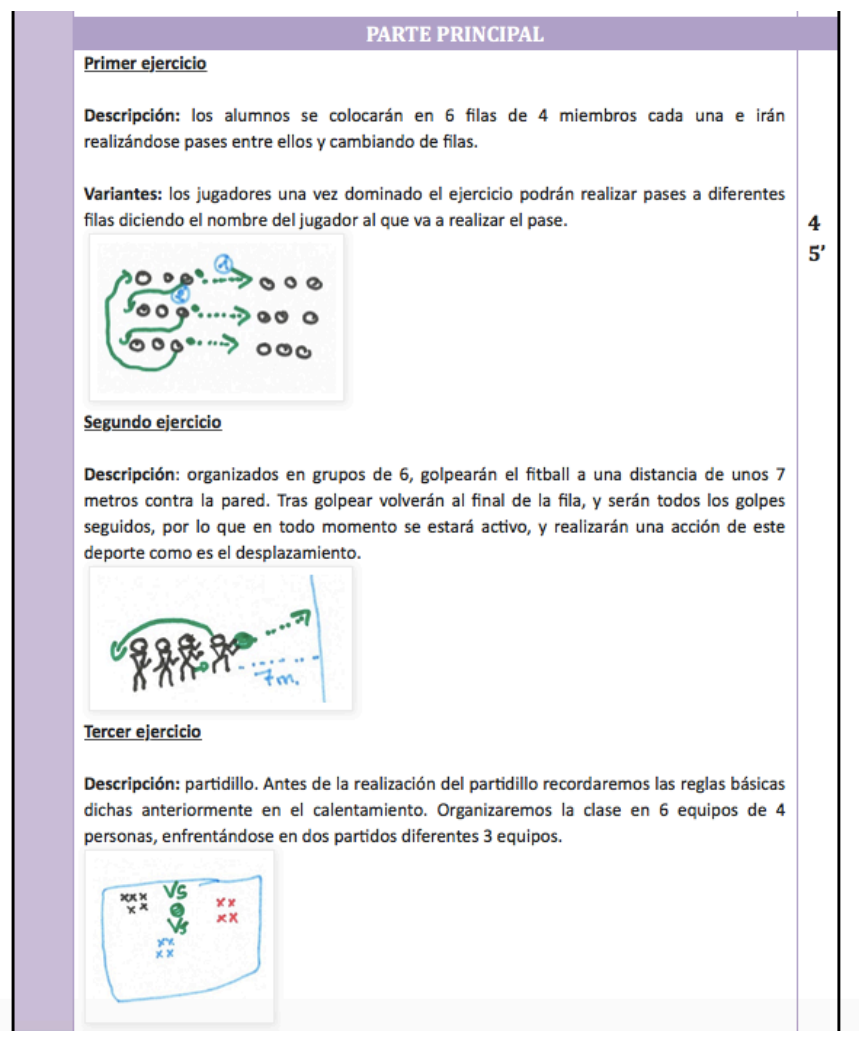

Imagen 1:Ejemplo de post de la asignatura de Juegos y deportes alternativos

El alumnado realizaba su post basándose en una serie de pautas facilitadas por el profesorado para escribirlo. Debía incluir un breve resumen del deporte alternativo sobre el que trabajaban; debían plasmar la sesión práctica que iban a realizar a través de enlaces a otras páginas web, imágenes, vídeos, infografías u otro tipo de recurso y; debían grabar y editar un vídeo de la sesión práctica (una vez realizada) aportando un comentario autocrítico sobre la sesión que habían realizado.

De la misma forma que la asignatura anterior, este blog se encuentra en fase de implementación al ser una asignatura del $2^{\circ}$ semestre de la titulación, por lo que no es posible extraer resultados concisos sobre él. No obstante, la acogida por parte del alumnado y el trabajo realizado hasta el momento parece satisfactorio. Al finalizar el blog se recogerán datos de forma cualitativa para su análisis a través de un último post de autovaloración.

En los tres blogs se realiza un último post enfocado a que el alumnado evalúe y reflexione críticamente sobre el trabajo desarrollado en él, es decir, que muestre cuál es su valoración del blog. Este post de reflexión final se utilizó para evaluar la experiencia por parte del profesorado y, concretamente, los comentarios que el alumnado realizó se utilizan para el análisis de datos (objetivo 9). 


\section{Análisis de los datos}

Dado las características de los datos recogidos, los comentarios que realizó el alumnado en blog para la pregunta de autoevaluación final, se utiliza una metodología cualitativa para su análisis. La investigación narrativa la define Bolívar (2002) como el análisis de los textos reconstruidos por las personas que plasman su experiencia, entendida y enmarcada en un contexto, que es capaz de otorgar significado a las vivencias personales a través de un proceso reflexivo. Bruner (1988) añade que la narrativa es una forma de construir la realidad desde la subjetividad ya que las palabras median, construyen y configuran la construcción social, que se torna realidad a través de la propia experiencia. El intercambio de subjetividades, en un proceso dialógico, se convierte en un modo de construir conocimiento.

Para analizar los discursos del alumnado optamos por un análisis categorial inductivo que permite emerger los significados y la realidad del uso del blog en nuestras asignaturas. Para comprender las vivencias en cuanto a los beneficios, limitaciones y mejoras que el alumnado considera que tiene este recurso didáctico, se analizaron los discursos agrupando las ideas que expresaban para, posteriormente, crear categorías de análisis.

Antes del análisis, se procedió a corregir las faltas de ortografía y se realizó una corrección gramatical para dar sentido a las ideas del alumnado de forma que se introdujeron puntos y comas para facilitar la compresión del texto.

\section{Resultados}

Los resultados que se presentan perteneces únicamente a una de las asignaturas (danza y expresión corporal) puesto que las otras dos, pertenecientes al segundo semestre, todavía están en activo.

En las asignaturas donde el blog está en proceso de elaboración, se pretenden recoger datos de forma cualitativa para su posterior análisis a través de post de autovaloración a la finalización de éstos. En el post de autovaloración de la asignatura de danza y expresión corporal se realizaron un total de 41 comentarios. Los nombres utilizados son pseudónimos.

En el contexto de la Universidad San Jorge y, concretamente en el grado en ciencias de la actividad física y el deporte, el uso de blogs es una herramienta innovadora puesto que el alumnado señala que no habían utilizado los blogs previamente ni en los ciclos anteriores (primaria, secundaria, bachillerato o ciclos de formación profesional) ni en ninguna otra asignatura del primer semestre de la titulación. En este sentido, la introducción de una herramienta educativa puede ser innovadora o no dependiendo del contexto por lo que la innovación tiene un componente experiencial clave. Incluso, dentro del mismo contexto puede ser innovadora para algunas personas pero no para otras.

"El blog de la asignatura de danza me ha parecido algo innovador, ya que solo lo hemos realizado en esta asignatura y, sinceramente, yo no me lo esperaba ya que, en todos los años que llevo estudiando, nunca había

(cc) EY-NC-ND 2018, Universitat Politècnica de València

Congreso IN-RED (2018) 
utilizado un blog para trabajar sobre documentos, vídeos, reflexiones que son de mucha información...” (Esteban).

\subsection{Desarrollo de habilidades}

El blog ha permitido desarrollar diversas habilidades instrumentales en el alumnado como son las habilidades de escritura, de búsqueda de información en la web y en otros soportes o la síntesis y resumen de ideas y textos de otros autores (objetivos 4 y 5). En este sentido, Agustín señala que:

"En mi caso, he aprendido a buscar información, en libros de la biblioteca de la universidad; y sobre todo en Internet, en las revistas digitales relacionadas con el deporte y la Educación Física, y libros que se podían leer a través de la vista previa"

Otra compañera expresa la misma idea señalando que (objetivo 6):

"El blog de la asignatura ha sido una herramienta de trabajo y aprendizaje muy positiva. No solo ha ayudado al alumnado a desarrollar competencias, como la autonomía o la expresión escrita, sino también al profesorado para que conozca el feedback que nosotros los alumnos, quienes al fin y al cabo realizamos los talleres y absorbemos la información que nos proporcionan, podemos plasmar a cerca de todos esos títulos propuestos en el blog” (Aitana).

Estudios previos indican que a través del blog el alumnado desarrolla habilidades de escritura y lectura, ya que enfrentarse a la redacción de un comentario o post para el blog requiere movilizar los aprendizajes y habilidades previas para conseguir la tarea. También, mejora la capacidad de buscar y reorganizar la información para darle sentido a las ideas y poder expresarlas a través de la producción escrita (Sim y Hew, 2010).

\subsection{Aprendizaje cooperativo}

Otro de los beneficios que se indica en el uso de blogs es que sirve como espacio para poder conocer las ideas de resto de compañeros (Coutinho, 2007). Esto favorece la creación de nuevas relaciones sociales con alumnado con el que habitualmente no se interactúa en las clases presenciales y, también, para el fortalecimiento de las relaciones sociales que ya existentes (Santos y Fernández-Rio, 2012). El progreso y la consecución del objetivo 8 quedó reflejado a través del registro personal del alumnado sobre la utilidad del blog para lograr un aprendizaje cooperativo.

"Otro aspecto a destacar sobre el blog ha sido la posibilidad de contrastar ciertas opiniones mediante los comentarios del resto de los compañeros de clase” (Juan) 
Algunos autores señalan que el intercambio de ideas es fundamental a la hora de establecer dinámicas de aprendizaje cooperativo (Huang et al.,2011). El alumnado valora positivamente poder conocer las ideas de los demás e incluso utilizarlas para poder construir a partir de ellas sus propias ideas, rebatirlas o construir de forma compartida el conocimiento sobre los temas de la asignatura (Coutinho, 2007). Esto se observa en palabras de Víctor:

"La reafirmación o discusión de lo que se presenta como comentario de cada participante, permite un debate que, por norma general, es enriquecedor para valorar aspectos positivos y negativos de los comentarios realizados”

No obstante, como el mismo Víctor señala: “[...] las opiniones y análisis han sido muy cerrados, con distintos puntos de vista, pero sin confrontación dialéctica entre ellos”. Sería necesario favorecer no sólo el poder acceder a las ideas de los demás, sino que se generara un debate que enriqueciera las posturas de todos los participantes. De este modo, además de generar dinámicas de aprendizaje cooperativo se favorece el sentido crítico puesto que los comentarios serán puestos a prueba por el colectivo de alumnos en un ciclo de feedback continuo.

\subsection{Evaluación del blog}

En relación a la evaluación del uso del blog y los aspectos de mejora (objetivo 9), el alumnado destaca entre los aspectos negativos que la posibilidad de hacer comentarios a los post debería poder realizarse durante los 15 días siguientes a su publicación. En cambio, se proponía que se pudieran realizar los comentarios en cualquier momento antes de la fecha de entrega del trabajo. En este sentido, un aspecto negativo que el alumnado encontraba era que los comentarios debían tener entre 500 y 700 palabras puesto que señalaban que en ocasiones era complicado llegar a ese número de palabras y, en otras, la extensión era insuficiente.

"Un aspecto negativo que he visto ha sido la duración de cada entrada, sólo duraban 2 semanas, para mi hubiera estado bien que se quedaran abiertas sin límite de duración” (Rafa).

"Por otro lado, había temas que se me hacían cortos, incluso llegando a 700 palabras, y otros que llegar a las 500 era un suplicio” (Carmen).

A pesar de esto, la introducción de nuevas herramientas y metodologías requieren de un tiempo de toma contacto y habituarse a las dinámicas para su aceptación. Dado que esta herramienta resulta novedosa para el alumnado es preciso fomentar su práctica y formarlos en ellas. La toma de decisiones en cuanto a las limitaciones que destaca el alumnado están basadas en experiencias previas realizadas en el uso de blogs y en las propias del 
profesorado participante, acumuladas a lo largo de la investigación previa y su uso en diferentes contextos educativos (véase Molina, Valencia-Peris y Gómez-Gonzalvo, 2016) y no a un criterio arbitrario por parte del profesorado.

\section{Conclusiones}

En este trabajo se constata que el uso del blog en las asignaturas ha alcanzado similares aprendizajes que en las experiencias previas tanto a nivel internacional como nacional. Se han encontrado que las percepciones e ideas del alumnado con respecto al uso del blog son similares a las encontrados por otros autores en cuanto al desarrollo de habilidades a través del blog, a las dinámicas de aprendizaje cooperativo que se desarrollan en él y que ha sido una propuesta de innovación en el contexto y para el alumnado al que estaba enfocado.

No obstante, como indican Molina, Valencia-Peris y Gómez-Gonzalvo (2016), los aspectos positivos de los blogs se dan en cualquier asignatura y contexto debido a las posibilidades de adaptación de esta herramienta. Estos autores identifican una serie de principios a la hora de utilizar el blog para garantizar cierta parte de éxito como son: informar al alumnado de qué se va hacer con el blog; cuál es el objetivo del blog en la asignatura y; otorgar al trabajo del blog un peso en la calificación acorde al trabajo que se va a realizar.

Como limitaciones cabe destacar que el proyecto se encuentra en fase de realización e implementación (queda por terminar el $2^{\circ}$ sementre del curso académico) y el objetivo de explorar una organización que permita progresar metodológicamente en su uso hacia la cesión de autonomía al alumnado todavía se encuentra en activo. Es por este motivo que los resultados aquí presentados son parciales y limitados por lo que deben ser tenidos en cuenta como provisionales.

\section{Referencias bibliográficas}

Antolín, L.; Molina, P.; Villamón, M.; Devís-Devís, J. y Pérez-Samaniego, V. (2011). “Uso de blogs en Ciencias de la Actividad Física y el Deporte”. @tic. revista d'innovació educativa, 7.

Bates, T. (2009). "Promesas y mitos del aprendizaje virtual en la educación postsecundaria”. En M. Castells (ed.). La sociedad red: una visión global (pp. 335-359). Madrid: Alianza Editorial.

Bolivar, A. (2002). “¿De nobis ipsis sile mus?”: Epistemología de la investigación biográfico-narrativa en educación. Revista Electrónica de Investigación Educativa, 4(1).

Bruner, J. (1988). Realidad mental, mundos posibles. Barcelona: Gedisa.

Coutinho, C. (2007). "Cooperative Learning in Higher Education using Weblogs: a study with undergraduate students of Education in Portugal". World multi-conference on systemics, cybernetic and informatics, 11 (1), 60-64. Disponible en 
http://repositorium.sdum.uminho.pt/bitstream/1822/6721/1/Webblogs.pdf?origin=publicati on_detail

Domingo, M. y Marqués, P. (2013). "Práctica docente en aulas 2.0 de centros de educación primaria y secundaria de España”. Píxel-Bit. Revista de Medios y Educación, 42, 115-128.

Gros, B y Suárez, C. (2016). Pedagogía Red. Una educación para tiempos de internet. Barcelona: Octaedro.

Harari, Y. (2017). Homo Deus. Madrid. Debate.

Huang, T. -C., Huang, Y. -M. y Yu, F. -Y. (2011). "Cooperative Weblog Learning in Higher Education: Its Facilitating Effects on Social Interaction, Time Lag, and Cognitive Load”. Educational Technology \& Society, 14 (1), 95-106.

Marqués, P. y Álvarez, I. (2014). "El Currículum bimodal como marco metodológico y para la evaluación: principios básicos y mejoras obtenidas en aprendizajes y rendimiento de los estudiantes”. Educar, 50(1), 149-166.

Molina, P.; Valencia-Peris, A. y Gómez-Gonzalvo, F. (2016). "Innovación en docencia universitaria: edublogs, evaluación formativa y aprendizaje colaborativo”. Profesorado. Revista de currículum y formación del profesorado, 20(2), 432-449.

Santos, L. y Fernández-Rio, J. (2012). "Uso pedagógico del blog: un proyecto de investigación-acción en la materia de educación física en educación secundaria”. Revista electrónica de tecnología educativa [EDUTEC], 42.

Sim, J.W.S. y Hew, K.F. (2010). "The use of weblogs in higher education settings: A review of empirical research”. Educational Research Review, 5, 151-163.

Sparkes, A. (1992). "Reflexiones sobre las posibilidades y los problemas del proceso de cambio en la Educación Física”. En Devís-Devís, J. y Peiró-Velert, C. (Ed.), Nuevas perspectivas curriculares en Educación Física: la salud y los juegos modificados (pp. 5166). Barcelona: Inde.

Wang, S.K. y Hsua, H.Y. (2008). "Reflections on using blogs to expand in-class discussion”. TechTrends, 52(3), 81-85.

Wang, Y.S., Lin, H.H. y Liao, Y.W. (2012). "Investigating de individual difference antecedents of perceived enjoyment in students' use blogging”. British journal of educational technology, 43(1), 139-152. 